\title{
Patient advocacy practice among Iranian nurses
}

\author{
Reza Negarandeh, Nahid Dehghan Nayeri
}

Nursing and Midwifery Care Research Centre, Faculty of Nursing and Midwifery, Tehran University of Medical Sciences, Tehran IRAN Author for correspondence: Nahid Nayeri e-mail: nahid.nayeri@gmail.com

\begin{abstract}
This cross-sectional study among nurses at the hospitals of the Tehran University of Medical Sciences aimed to investigate the extent of involvement in patient advocacy practice among nurses. Participants ( $n=330$ ) were selected using proportionate systematic random sampling. Data were gathered through patient advocacy questionnaires and analysed using descriptive/inferential statistics. The average of nurses' patient advocacy practice was 3.848+ 0.304 (possible range 1-5). One third of the participant nurses had high scores in practising patient advocacy, two thirds of them had scores in the medium range and $0.6 \%$ of nurses had low scores. The majority of nurses (83.5\%) declared their commitment to patient advocacy as high, and $42.5 \%$ believed they had enough ability to practise advocacy. Half of the participants claimed to have faced problems following advocacy practices. The problems that most participants reported included conflict with colleagues and/or superiors, receiving oral/written admonitions, and shift and/or ward changes. Patient advocacy scores were significantly higher among female nurses, head nurses, and those who had more commitment and ability for patient advocacy $(p<0.001)$. In contrast, nurses who worked in more than one hospital simultaneously due to financial need $(p=0.048)$, and experienced conflict with superiors $(p=0.047)$ scored less than others. The nurses in our study carried out advocacy at acceptable levels and adopted it as part of their own professional role. Nurses tended to advocate for patients, but they faced many challenges in this.
\end{abstract}

\section{Introduction}

Nursing advocacy as a core value is a key component of the professional nursing role (1). In the nursing literature, clients' vulnerability is explained as the main reason for advocacy action (2). Many studies have shown that medicine has the potential to restrict the autonomy of individuals, disabling and dominating over people through bureaucracy, scientific expertise and technology (3). The majority of patients exhibit various degrees of vulnerability to such domination by virtue of their illness, cultural, economic or educational background, personality, and previous experiences with the healthcare system.

According to Vaartio and Leino-Kilpi (4), advocacy is an integral part of the role of professional healthcare practitioners in promoting the well-being of patients. However, nurses show greater commitment than other healthcare professionals in involving themselves in patient advocacy $(5,6)$. It is suggested that nurses' proximity to patients and the quality of the nurse-patient relationship prompt them to take on patient advocacy (7).

The International Council of Nurses (ICN) introduced the concept of advocacy in the code of ethics for nurses (4). The ICN Code of Ethics for Nurses (8:4) states that "in providing care, the nurse promotes an environment in which the human rights, values, customs and spiritual beliefs of the individuals, families and communities are respected". The ICN calls on nurses and nursing organisations to promote advocacy as a key nursing role (9). Mallik (2) believes that patient advocacy is a traditional role for nurses who are in the best position, within the healthcare team, to act as patients' advocates. Nurses are considered to be in the frontline of care, and to have a good grasp of the issues involved. Mallik also believes that nurses and patients can be partners in advocacy. Moreover, international healthcare reforms emphasising human rights have increased the significance of nursing advocacy (10).

While patient advocacy is considered to be a core component for nursing practice, its application in practice by Iranian nurses is not so clear. Negarandeh et al (11) conducted a study to explore patient advocacy practice among Iranian nurses, using grounded theory. Based on their findings, they recommended that quantitative research be conducted to examine advocacy practice by nurses and identify the barriers and facilitators in the process, if any.

This study aimed to investigate patient advocacy practices and the correlation with personal characteristics among hospital nurses in the Tehran University of Medical Sciences, Iran.

\section{Review of literature}

According to Gaylord and Grace (12), nurses feel an ethical obligation to advocate on behalf of their patients. Moreover, they frequently describe their decisions and actions on behalf of patients as 'being a patient advocate' $(13,14)$. Advocacy has been described in different ways. It can refer to protecting, mediating, and acting for patients (15); safeguarding patients from incompetence or misconduct of other healthcare professionals (16); asking doctors questions on behalf of patients, giving information, and maintaining patients' privacy during nursing procedures (17); helping patients to obtain the necessary healthcare, assuring quality of care, defending patients' rights, and serving as a liaison between patients and the healthcare system (18); safeguarding patients' autonomy, 
and championing social justice in the provision of healthcare (19); and acting on behalf of patients $(17,19)$.

According to Smith (20), a public survey in the US, regarding patient advocacy as seen from the patient's perspective, found that $95 \%$ of patients interviewed agreed that compassion plays a key role in the healing process. Observing dignity, showing interest in patients' questions, paying attention to their needs and respecting cultural/racial/religious needs were described as dimensions of compassion. Davis et al (21) in their study examined what nurses thought about their patient advocacy role in the Japanese health system. They found that $79 \%$ of participants responded positively to the question: "To what extent do you act as a patient advocate?" They also found that nurses advocate for patients in two ways: by providing information to patients and their families, and protecting patients from acts of misconduct. It must be noted that the latter action can disturb nurse-physician or physician-patient/ family relationships.

Ware et al (22) found, in a study conducted by the American Society for Pain Management Nursing Research Committee on nurses' advocacy activities in pain management, that the majority of nurses reported being involved in personal advocacy to protect the patient. In this activity, they said they even confronted physicians when necessary.

Kubsch et al (23) investigated factors influencing the practice of advocacy from the nurses' perspective. 52 nurses responded to the questionnaire developed by researchers. The findings indicated that there was limited exercise of patient advocacy among nurses. Boyle (24), in a phenomenological study, investigated patient advocacy in the perioperative setting. He found that four main themes emerged from 33 interviews with nurses: protection, communication/ giving voice, doing, and comfort and caring.

Nurses usually experience both fulfilment and frustration in the advocacy role (25). When their efforts are effective and the aims of advocacy are met, they experience positive feelings such as happiness, calmness, and satisfaction. Effective advocacy also saves patients from harm. O'Connor and Kelly (9) believe that successful patient advocacy actions bring about positive consequences for nurses, such as professional satisfaction, self-confidence, self-esteem, and a sense of personal integrity. However, sometimes, the attempts of nurses to advocate on behalf of patients fail. In this situation, the aim is not met, and consequently, patients remain vulnerable. Moreover, nurses face some risks and negative feedback from colleagues, physicians, and their institutions. According to Hanks (25), nurses may find that nursing advocacy can threaten their careers. Some authors discuss the practice of nursing advocacy as entailing risks and that can result in frustration and anger $(2,18)$. In their working environments, nurses can be confronted with punishments such as demotion in the institutional hierarchy, and being labelled by their peers as instigators, troublemakers or whistleblowers. The act of advocating for patients can result in the disruption of relationships with peers, other healthcare workers, and the institutional administration.
Rushton (26) notes that effective leadership, open communication patterns, collaborative problem-solving methods, compatibility of values and philosophy among various healthcare team members, and procedural safeguards such as ethics committees facilitate advocacy and promote patient outcomes. Snowball (14) asserts that a therapeutic relationship is central to advocacy. O'Connor and Kelly (9) state that the ability to advocate is based on sound nursing knowledge and expertise.

During the three last decades, patient advocacy has been emphasised as an important role for nurses in nursing literature. However, Davis et al (21) report that patient care and protection could become secondary to self-protection, without the support of the nursing leadership for advocacy. Advocacy for clients is still a risky role for nurses. It can be hindered by real or perceived legal constraints, societal values and factors, organisational culture, nursing status within the healthcare system, and political and economic constraints (26). In a study by Negarandeh et al (11) to investigate the barriers and facilitators influencing the role of advocacy among Iranian nurses, the participants felt that they did not receive any support for advocacy action from their managers. They also reported that powerlessness, the lack of a national code of ethics, and the risks of advocacy acted as barriers to patient advocacy.

Our knowledge of nursing advocacy is based more on theoretical than on empirical works. Moreover, the majority of studies on nursing advocacy are qualitative studies. (27) We could not find any studies that included a quantitative study of nurses' patient advocacy practices in Iran. Recent articles on this subject have focused on clarification of the concept, and developing theories, models and instruments. There are few quantitative studies $(1,19,28)$.

\section{Methods}

\section{Design and sample}

This cross sectional study was conducted in the hospitals of the Tehran University of Medical Sciences in 2010. Nurses with at least one year's full-time work experience in clinical practice and having an associate degree, or higher educational preparation in nursing, were included in the study. We obtained completed questionnaires from 330 out of 2,500 registered nurses working in 14 hospitals, using systematic randomised sampling according to the number of nurses in each hospital. A researcher was present at the hospital units and delivered the questionnaires to the participants. The nurses were informed of the purpose and process of the study. Completion of the questionnaires by the nurses was considered as implied consent of the participants. We continued the sampling process until the needed sample size was obtained. Thus, if the selected nurse did not wish to participate or complete the delivered questionnaire, we randomly selected the next nurse from the list. The refusal rate was $3 \%$. 


\section{Study design}

A questionnaire was developed based on the literature and included the results of a previously conducted grounded theory study on nursing advocacy $(11,29)$. The questionnaire consisted of two parts. In the first part (16 items), 13 items assessed the participants' personal characteristics including their age, gender, marital status, educational level, working shift, work experience, position, whether they worked overtime on a regular basis, and whether they had work experience in another hospital. Two Likert-scale items assessed the commitment of nurses and their ability for patient advocacy practice. One item assessed the risks that patient advocacy participants reported encountering. Nurses were asked to rate their own commitment and ability for patient advocacy. They were also questioned as to the consequences they faced in their work environment as a result of being patient advocates. The second part consisted of 40 items to assess the patient advocacy practices of nurses. Some items included in the questionnaire were: giving the patient information, clarifying doctors' comments for patients, answering patients' questions, supporting patients' decisions, helping patients to obtain needed healthcare, devoting time to listen to patients, protecting patients' privacy, and acting on their behalf. Each question used a five-point Likert scale, extending from 'never' to 'always'. The possible range of scores for patient advocacy practices was from 40 to 200. These acquired scores were then divided by the number of questions, so that the range of obtained scores amounted to between one and five. Thus, the average scores of $1-2,3$, and $4-5$ were considered low, medium, and high, respectively.

The content validity of the questionnaire was established through review by an expert panel. Changes were made based on the experts' opinions. The content validity index (CVI) was calculated for each item of the questionnaire. Those with an item-CVI ( I-CVI) score of more than 0.80 were kept in the final questionnaire. Reliability was determined using the test and retest methods, with a two-week interval, among a sample of 15 nurses $(r=0.86)$.

\section{Ethical considerations}

The research proposal was approved by the university research council and research ethics committee. We stated to the participants that by completing the questionnaire they were giving their consent for us to use the data for analysis and report the study's findings. We also stated that their identities would not be revealed.

\section{Data analysis}

The data were analysed using descriptive and inferential statistics such as independent t-test and one-way variances (ANOVA) and were presented in frequency tables and crosstabs. A p-value less than 0.05 was considered as significant.

\section{Results}

Data analysis revealed that the participants' ages ranged from 22 to 55 years with mean and standard deviation (35.02 \pm 7.46). Participants' personal characteristics were presented in
Table 1. The length of the participants' work experience ranged from one to 30 years, with a mean of 11.48 years $(S D=7.25)$. In addition, 255 nurses worked as staff nurses (78.5\%), 55 of the participants held head nurse positions (16.9\%), and 15 nurses worked as supervisors (4.6\%). More than half of the nurses (57.7\%) worked in night shifts. Although almost one third of the participant nurses did not work overtime (29\%), the rest (71\%) had overtime scheduling every month. While $43.3 \%$ of the participants did less than 50 hours overtime in a month, $24.7 \%$ worked between 50 to 100 hours overtime, and $3 \%$ worked more than 100 hours overtime per month. Also, 32 participants $(9.8 \%)$ worked in other hospitals simultaneously as nursing staff. Only $33.7 \%$ of nurses had attended continuing education programmes regarding patient advocacy.

Table 1: Participants' demographic characteristics

\begin{tabular}{|c|c|c|c|}
\hline & & Frequency & Valid percentage (\%) \\
\hline \multirow{5}{*}{ Age } & $22-30$ & 110 & 37 \\
\hline & $31-40$ & 124 & 41.8 \\
\hline & $41-53$ & 63 & 21.2 \\
\hline & Missing & 33 & - \\
\hline & Sum & 330 & 100 \\
\hline \multirow{4}{*}{ Gender } & Male & 40 & 12.2 \\
\hline & Female & 288 & 87.8 \\
\hline & Missing & 2 & - \\
\hline & Sum & 330 & 100 \\
\hline \multirow{5}{*}{$\begin{array}{l}\text { Marital } \\
\text { status }\end{array}$} & Unmarried & 138 & 42.3 \\
\hline & Married & 187 & 57.4 \\
\hline & Divorced & 1 & 0.3 \\
\hline & Missing & 4 & - \\
\hline & Sum & 330 & 100 \\
\hline \multirow{5}{*}{ Education } & $\begin{array}{l}\text { Associate } \\
\text { degree }\end{array}$ & 5 & 1.5 \\
\hline & $\begin{array}{l}\text { Baccalaureate } \\
\text { degree }\end{array}$ & 309 & 94.5 \\
\hline & Master's degree & 13 & 4 \\
\hline & Missing & 3 & - \\
\hline & Sum & 330 & 100 \\
\hline
\end{tabular}

$32.4 \%$ of nurses obtained high scores in practising patient advocacy; $67.0 \%$ of the nurses were moderately successful and $0.6 \%$ of nurses achieved low scores on the patient advocacy scale (Table 2).

According to the results, the majority of participants reported that they helped patients in their decision making (74.5\%), 
knew medical diagnosis (81\%), and built appropriate relationships with healthcare team members (86\%). In addition, more than $95 \%$ of the participants in this study believed that they respected patients' beliefs and values, and considered patients' privacy and confidentiality throughout their nursing care. On the other hand, only $56.4 \%$ of participants reported that they interceded to stop mistakes occurring in clients' care. $55 \%$ of the participants claimed to have put in great effort to restore equity in the healthcare system.

\section{Table 2: Frequency distribution of patient advocacy} practices

\begin{tabular}{|l|c|c|}
\hline Patient advocacy practices & Frequency & Percentage (\%) \\
\hline Low & 2 & 0.6 \\
\hline Moderate & 221 & 67.0 \\
\hline High & 107 & 32.4 \\
\hline Sum & 330 & 100 \\
\hline \multicolumn{2}{|r|}{$\begin{array}{r}\text { Mean (Standard deviation) 3.848 (0.304) } \\
\text { [95\% Cl 3.832 to 3.865] }\end{array}$} \\
\hline
\end{tabular}

Analysis of the participants' responses to two questions about the nurses' commitment and capacity for patient advocacy showed that the majority of nurses (83.5\%) declared their commitment to patient advocacy to be high; $42.5 \%$ of them believed that their capacity for patient advocacy was substantial (Table 3 ).

Table 3: Commitment and capacity of nurses for patient advocacy

\begin{tabular}{l|c|c}
\hline Degree of commitment & Frequency & Valid percentage (\%) \\
\hline Low & 2 & 0.6 \\
Moderate & 52 & 15.9 \\
High & 274 & 83.5 \\
\hline Missing & 2 & - \\
\hline Sum & 330 & 100.0 \\
\hline Degree of capacity & Frequency & Valid percentage (\%) \\
\hline Low & 25 & 7.7 \\
Moderate & 162 & 49.8 \\
High & 138 & 42.5 \\
\hline Missing & 5 & 100.0 \\
\hline Sum & 330 & - \\
\hline
\end{tabular}

In addition, $50.3 \%$ of nurses experienced problems resulting from their patient advocacy attempts. The most common problems were: conflicts with colleagues, conflicts with superiors, receiving oral and written admonition, and transfers from their shifts or wards.
Table 4: Correlation of personal characteristics with patient advocacy practices score

\begin{tabular}{|c|c|c|c|c|c|}
\hline & & Frequency & Mean & SD & Significance \\
\hline \multirow{4}{*}{ Gender } & Male & 40 & 3.689 & 0.313 & \multirow{2}{*}{$p<0.001$} \\
\hline & Female & 288 & 3.870 & 0.297 & \\
\hline & Missing & 2 & & & \\
\hline & Sum & 330 & & & \\
\hline \multirow{5}{*}{ Position } & Nurse & 255 & 3.816 & 0.296 & \multirow{3}{*}{$p<0.001$} \\
\hline & $\begin{array}{l}\text { Head } \\
\text { nurse }\end{array}$ & 55 & 3.976 & 0.299 & \\
\hline & Supervisor & 15 & 3.941 & 0.362 & \\
\hline & Missing & 5 & & & \\
\hline & Sum & 330 & & & \\
\hline \multirow{5}{*}{$\begin{array}{l}\text { Commitment } \\
\text { to patient } \\
\text { advocacy }\end{array}$} & Low & 2 & 3.075 & 0.601 & \multirow{3}{*}{$p<0.001$} \\
\hline & Medium & 52 & 3.656 & 0.279 & \\
\hline & High & 274 & 3.892 & 0.286 & \\
\hline & Missing & 2 & & & \\
\hline & Sum & 330 & & & \\
\hline \multirow{5}{*}{$\begin{array}{l}\text { Capacity } \\
\text { for patient } \\
\text { advocacy }\end{array}$} & Low & 25 & 3.649 & 0.454 & \multirow{3}{*}{$\mathrm{p}<0.001$} \\
\hline & Medium & 162 & 3.802 & 0.264 & \\
\hline & High & 138 & 3.936 & 0.292 & \\
\hline & Missing & 5 & & & \\
\hline & Sum & 330 & & & \\
\hline \multirow{4}{*}{$\begin{array}{l}\text { Working in } \\
\text { more than } \\
\text { one hospital }\end{array}$} & Yes & 32 & 3.752 & 0.337 & \multirow{2}{*}{$p=0.048$} \\
\hline & No & 293 & 3.864 & 0.300 & \\
\hline & Missing & 5 & & & \\
\hline & Sum & 330 & & & \\
\hline \multirow{4}{*}{$\begin{array}{l}\text { Experiencing } \\
\text { conflict with } \\
\text { superiors }\end{array}$} & Yes & 82 & 3.789 & 0.317 & \multirow{2}{*}{$p=0.047$} \\
\hline & No & 241 & 3.867 & 0.300 & \\
\hline & Missing & 7 & & & \\
\hline & Sum & 330 & & & \\
\hline
\end{tabular}

The mean of patient advocacy practices scores showed significant relationships with gender, position, commitment to patient advocacy and capacity for patient advocacy $(p<0.001)$. In other words, patient advocacy scores were significantly higher among female nurses, head nurses, and those with more commitment and capacity for patient advocacy. Working in more than one hospital $(p=0.048)$, and experiencing conflict with superiors $(p=0.047)$ were also factors significantly related to patient advocacy. This means that nurses who worked in two hospitals simultaneously, and those who experienced conflict with superiors scored less in patient advocacy than 
others. The mean of patient advocacy practice scores was different among male and female nurses (gender), nurses who work as staff nurses, head nurses and matrons (position), and built on experiencing or not experiencing conflict with superiors. We used a linear term test for degree of commitment and capacity for patient advocacy because of their ordinal scale. The results revealed a trend $(p<0.001)$ between the two mentioned variables and patient advocacy practices. So having a higher degree of commitment and a greater capacity for patient advocacy acted as facilitating factors for improvement in patient advocacy (Table 4).

\section{Discussion}

Nurses who participated in this study showed high (32.4 $\%)$, medium (67.0 \%) and low (0.6\%) participation in patient advocacy practices. This finding is similar to the findings of Ware et al (22) that a majority of nurses are active in patient advocacy. In addition to this, Davis et al (21) found that $79 \%$ of Japanese nurses have seen themselves as patient advocates. In contrast to this, Kubsch et al (23) found the participant nurses were involved in moral-ethical advocacy, meaning that nurses upheld the patient's values in decision-making, more often than they participated in legal, political, spiritual or substitutive advocacy (mean 2.83, possible range 1-5). From this, Kubsch et al concluded that nurses' participation in patient advocacy was limited. It should additionally be noted that this finding is also comparable with the findings of the qualitative study conducted by Negarandeh et al $(11,29)$ in Iran. This finding is important because the ICN code of ethics also emphasises promotion of patient advocacy among nurses (8).

Participants in this study showed a strong commitment to nursing advocacy. A similar finding was reported in the study by Davis et al (21). O'Connor and Kelly (9) state that professional responsibility is a key trigger for advocacy. According to Bernal (30), "the primary obligation is to patients, rather than to physicians or hierarchies within the hospitals". Negarandeh et al (11) in their qualitative study report that all participants perceive advocacy as one of their roles and obligations. Moreover, the participants believed that patients are vulnerable in hospitals.

As mentioned earlier, most of the participants in our study were committed to patient advocacy, but only half of them were able to achieve it. This inconsistency can be justified by the presence of numerous barriers to patient advocacy in hospital settings. Hanks (27) also writes that several studies report the work setting as an influential factor in advocacy. Hellwig et al (18) and Kohnke (31) believe that the greatest obstacle to advocacy is the healthcare system itself, because client advocacy is basically in conflict with the culture of the hospital system. Traditionally, physicians have had a dominant position in Iran's healthcare system. Simultaneously, the majority of nurses who work in public hospitals are novices with less than two years of work experience. As a result, they cannot communicate confidently with patients or advocate for them. Patients and nurses are therefore easily placed in difficult situations. Hence, if advocacy practices are to be promoted among nurses, the organisational culture needs to be transformed into a supportive one.

According to our findings, $50.3 \%$ of the participants were confronted with problems during their patient advocacy practice. The most common types of problems revealed in the study were: conflicts with colleagues, conflicts with superiors, receiving oral or written admonition, and being transferred from their work shift or ward. Similar findings are reported by Davis et al (21): Japanese nurses take risks in practising patient advocacy. Some of the nurses in their study feared that the reported behaviour could damage their relationship with the physician, patients, or their families. Advocacy may result in loss of status, or job, or place nurses in indirect $(2,13)$ or direct conflict with the organisation (13). According to Hanks (25), nurses can experience both a sense of achievement and frustration with the nurse-advocate role. It was seen that encountering complications and failing in advocacy could hinder nurses from advocating for their patients. It is therefore not reasonable to expect nurses to maintain their advocacy role without changing the current unfavourable environment.

Based on the results of our study, the mean patient advocacy practice scores differed between male and female nurses. It seems female nurses involve themselves more in patient advocacy than their male colleagues. Staff nurses were less involved in patient advocacy than head nurses and supervisors. Obviously authority and accountability are determinant factors for patient advocacy. Several studies have also shown that having power, responsibility, and capacity can improve patient advocacy practices $(9,13)$. Being responsible for patients leads nurses to do their best for patients. Nurses who experience conflict with superiors were less involved in advocacy. Nurses' earnings are low in Iran, therefore Iranian nurses often work in more than one hospital. Furthermore, patient advocacy is still a risky role for nurses. In these circumstances, poor financial status and/or experience of conflict with superiors can inhibit nurses in their advocacy practices. Sellin considers problems related to job security and management conflicts to be strong barriers to nursing advocacy (13). We also found that there is a link between degree of commitment and level of capacity in the practice of patient advocacy. Thus, having a higher degree of commitment and higher level of capacity for patient advocacy improved performance and acted as facilitating factors for patient advocacy.

\section{Conclusion}

The results of our study reveal that Iranian nurses have an inclination towards implementing patient advocacy. However, their contribution as patient advocates is not acknowledged in the Iranian healthcare system and they encounter multiple obstacles. These limitations in Iranian hospitals can lead to poor quality and negligence in care. Nurses should adopt an advocacy role and act on behalf of patients because they interact more closely with patients than other healthcare staff. However, it was noted that the nurses' sense of responsibility for patients motivates them to act as patient advocates in spite of the existing obstacles. 
Acknowledgement The authors would like to thank the Tehran University of Medical Sciences (TUMS) which funded and supported this study under Grant no. 6537.

\section{References}

1. Hanks RG. Development and testing of an instrument to measure protective nursing advocacy. Nurs Ethics. 2010 Mar;17(2):255-67.

2. Mallik, M. Advocacy in nursing - a review of the literature. J Adv Nurs. 1997 Jan; 25(1): 130-8.

3. Hewitt, J. A critical review of the arguments debating the role of the nurse advocate. J Adv Nurs. 2002 Mar; 37(5):439-45.

4. Vaartio $H$, Leino-Kilpi $H$. Nursing advocacy--a review of the empirical research 1990-2003. Int J Nurs Stud. 2005 Aug;42(6):705-14.

5. Wheeler P. Is advocacy at the heart of professional practice? Nurs Stand. 2000 May 24-30; 14(36):39-41.

6. Grace, P. J. Professional advocacy: widening the scope of accountability. Nurs Philosophy. 2001;2(2):151-62.

7. Mahlin M. Individual patient advocacy, collective responsibility and activism within professional nursing associations. Nurs Ethics. 2010 Mar; 17(2):247-54.

8. International Council of Nurses (ICN). Code of Ethics for Nurses [Internet]. 2006 [cited 2011 Jul 4]. Available from: www.icn.ch/about-icn/code-ofethics-for-nurses/

9. O'Connor T, Kelly B. Bridging the gap: a study of general nurses' perceptions of patient advocacy in Ireland. Nurs Ethics. 2005 Sep 12(5):453-67.

10. Jugessur T, lles IK. Advocacy in mental health nursing: an integrative review of the literature.J Psychiatr Ment Health Nurs. 2009 Mar; 16(2):18795.

11. Negarandeh R, Oskouie F, Ahmadi F, Nikravesh M, Hallberg IR. Patient advocacy: barriers and facilitators BMC Nurs. 2006 Mar1;5:3.

12. Gaylord N, Grace P. Nursing advocacy: an ethic of practice. Nurs Ethics. 1995 Mar; 2(1):11-8.

13. Sellin SC. Out on a limb: a qualitative study of patient advocacy in institutional nursing. Nurs Ethics. 1995 Mar;2(1):19-29.

14. Snowball J. Asking nurses about advocating for patients:'reactive' and 'proactive' accounts. J Adv Nurs. 1996 Jul; 24(1): 67-75

15. Nelson ML. Advocacy in Nursing.Nurs Outlook. 1988 May-Jun;36 (3):13641.
16. Schroeter K. Ethical perception and resulting action in perioperative nurses. AORN J 1999 May; 69 (5):991-1002.

17. Breeding J.Turner de S. Registered nurses' lived experience of advocacy within a critical care unit:A phenomenological study.Aust Crit Care. 2002 Aug;15(3):110-7.

18. Hellwig SD, Yam M, DiGiulio M. Nurse case managers' perceptions of advocacy: A phenomenological inquiry. Lippincotts Case Manag, 2003Mar-Apr; 8(2):53-63; quiz 64-5.

19. Bu X, Jezewski MA. Developing a mid range theory of patient advocacy through concept analysis. J Adv Nurs. 2007 Jan;57(1):101-10.

20. Smith AP, Patient advocacy: roles for nurses and leaders. Nurs Econ. 2004 Mar-Apr;22(2):88-90.

21. Davis AJ, Konishi E, Tashiro M. A pilot study of selected Japanese nurses' ideas on patient advocacy. Nurs Ethics. 2003 Jul;10(4):404-13.

22. Ware L, Bruckenthal P, Davis GC, O'Conner-Von SK. Factors that influence patient advocacy by pain management nurses: results of the American society for pain management nursing survey. Pain Manag Nurs. 2011Mar;12(1):25-32. Epub 2010 Jul 24.

23. Kubsch SM, Sternard MJ, Hovarter R, Matzke V. A holistic model of advocacy:factors that influence its use.Complement Ther Nurs Midwifery. 2004 Feb;10(1):37-45.

24. Boyle HJ.Patient advocacy in the perioperative setting.AORNJ.2005Aug; 82(2):250-62.

25. Hanks RG. The lived experience of nursing advocacy. Nurs Ethics. 2008 Jul; 15(4):468-77.

26. Rushton $\mathrm{CH}$. Creating an ethical practice environment: a focus on advocacy. Crit Care Nurs Clin North Am. 1995 Jun; 7(2):387-97.

27. Hanks, R. G. The medical-surgical nurse perspective of advocate role. Nurs Forum. 2010 Apr-Jun; 45(2):97-107.

28. Bu X, Wu YW. Development and psychometric evaluation of the instrument: attitude toward patient advocacy. Res Nurs Health. 2008 Feb;31(1):63-75.

29. Negarandeh R, Oskouie F, Ahmadi F, Nikravesh M.The meaning of patient advocacy for Iranian nurses. Nurs Ethics. $2008 \mathrm{Jul}$; 15(4):457-67.

30. Bernal EW. The nurse as patient advocate. Hastings Cent Rep. 1992 JulAug; 22(4):18-23.

31. Kohnke M F. The nurse as advocate. Am J Nurs. 1980 Nov; 80(11):203840.

\section{Change in IJME mailing address}

Our address for subscription and correspondence has changed to Indian Journal of Medical Ethics c/o Survey No. 2804-5, Aaram Society Road, Vakola, Santacruz (E), Mumbai 400055. e-mail:ijmemumbai@gmail.com / ijme.editorial@gmail.com 